Pacific Journal of Mathematics

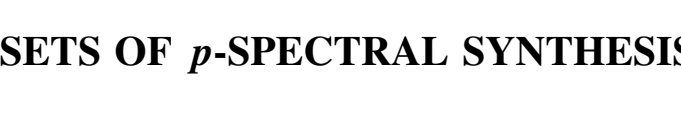




\title{
SETS OF $p$-SPECTRAL SYNTHESIS
}

\author{
Walter R. Bloom
}

Let $G$ be a Hausdorff locally compact Abelian group, $\Gamma$ its character group. Certain closed subsets of $\Gamma$ are introduced, these being closely related to sets of spectral synthesis for $L^{1}(G)^{\wedge}$. Some properties and examples of these sets are discussed, and then a Malliavin-type result is obtained.

In general we follow the notation used in [1]. We shall let $\lambda, \theta$ denote Haar measures on $G, \Gamma$ respectively, chosen so that Plancherel's theorem holds.

1. The definition and some properties of $S_{p^{-}}$and $C_{p}$ - sets.

Definition 1.1. Let $\Xi$ be a closed subset of $\Gamma$. We shall call $\Xi$ an $S_{p}$-set $(p \in[1, \infty))$ if, given $\epsilon>0$ and $f \in L^{1} \cap L^{p}(G)$ such that $\hat{f}$ vanishes on $\Xi$, there exists $g \in L^{1} \cap L^{p}(G)$ such that $\hat{g}$ vanishes on a neighbourhood of $\Xi$ and $\|f-g\|_{p}<\epsilon$. If such a $g$ can be found of the form $h * f$, where $h \in L^{\prime}(G)$ and $\hat{h}$ vanishes on a neighbourhood of $\Xi$, then $\Xi$ will be called a $C_{p}$-set. We also define $S_{\infty}$ - and $C_{\infty}$-sets as above, with $f, g$ in $L^{1} \cap C_{0}(G)$ (rather than $L^{1} \cap L^{\infty}(G)$ ).

Since, by [1], (33.12), $L^{1}(G)$ admits a bounded positive approximate identity $\left\{u_{i}\right\}_{i \in I}$ such that for each $i \in I, u_{i} \in L^{1} \cap C_{0}(G)$ and $\operatorname{supp}\left(\hat{u}_{i}\right)$ is compact, it follows (see [1], (32.33) (b) and (32.48) (a)) that we can (and shall) assume in Definition 1.1 that $f, g, h \in L^{1} \cap C_{0}(G)$, where $\operatorname{supp}(\hat{f})$ is compact and both $\operatorname{supp}(\hat{g})$ and $\operatorname{supp}(\hat{h})$ are compact and disjoint from $\Xi$ $(p \in[1, \infty])$.

Clearly every $C_{p}$-set is an $S_{p}$-set. For the case $p=1$ we just have the familiar $S$-set and $C$-set; see [3], 7.2.5 (a) and 7.5.1 respectively.

For $f \in L^{\infty}(G)$ the spectrum (written $\Sigma(f)$ ) will be defined as in [1], (40.21). For $f \in L^{p}(G)(p \in[1, \infty))$, we define its spectrum by

$$
\Sigma(f)=\cup\left\{\Sigma(\phi * f): \phi \in C_{00}(G)\right\}
$$

It is easily proved that for $f \in L^{\prime}(G), \Sigma(f)=\operatorname{supp}(\hat{f})$.

Given $\Xi \subset \Gamma$, we write

$$
L \stackrel{p}{E}(G)=\left\{f \in L^{p}(G): \Sigma(f) \subset \Xi\right\} .
$$


We now have the following characterisation of $S_{p^{-}}$and $C_{p}$-sets:

THEOREM 1.2. Let $p \in[1, \infty)$ and suppose $\Xi$ is a closed subset of Г. Then

(a) $\Xi$ is an $S_{p}$-set if and only if for all $l \in L L_{\Xi}^{P^{\prime}}(G)$ and for all $f \in L^{1} \cap C_{0}(G)$ such that $\operatorname{supp}(\hat{f})$ is compact and $\hat{f}$ vanishes on $\Xi$, we have $l * f=0$;

(b) $\Xi$ is a $C_{p}$-set if and only if for all $f \in L^{1} \cap C_{0}(G)$ such that $\operatorname{supp}(\hat{f})$ is compact and $\hat{f}$ vanishes on $\Xi$, and for all $l \in L^{p^{\prime}}(G)$ such that $l * f \in L_{\underline{\Xi}}^{p^{\prime}}(G)$, we have $l * f=0$.

This result is known for the case $p=1$ (see [2], Chapter 7, 1.2 and 4.9). The proof is standard, and we shall not include it.

It is easy to adapt the proof of [3], Theorem 7.5.2 to give:

TheOREM 1.3. Let $p \in[1, \infty]$. Then

(a) every one-point subset of $\Gamma$ is a $C_{p}$-set in $\Gamma$;

(b) finite unions of $C_{p}$-sets in $\Gamma$ are $C_{p}$-sets in $\Gamma$;

(c) if the boundary of a closed set $\Xi$ is a $C_{p}$-set, so is $\Xi$;

(d) if $\Xi$ is a closed subset of a closed subgroup $\Lambda$ of $\Gamma$, if $\partial_{\Lambda}(\Xi)$ is the boundary of $\Xi$ relative to $\Lambda$, and if $\partial_{\Lambda}(\Xi)$ is a $C_{p}$-set in $\Gamma$ then $\Xi$ is also a $C_{p}$-set in $\Gamma$;

(e) each closed subgroup of $\Gamma$ is a $C_{p}$-set in $\Gamma$.

For $p \in[1,2)$ it is not known whether the notions of $C_{p}$-set and $S_{p}$-set are identical (it appears in Theorem 2.1 that every closed set is a $C_{p}$-set for $p \geqq 2$ ). Furthermore we cannot say whether the union of two $S_{p}$-sets is itself an $S_{p}$-set. We can however obtain two partial results in this direction. Both these results (Theorem 1.4 (a), (b)) are known for the case $p=1$ (see [2], Chapter 2, 7.5).

THEOREM 1.4. (a) Suppose $\Xi=\Xi_{1} \cup \Xi_{2}$, where $\Xi_{1}$ and $\Xi_{2}$ are disjoint closed subsets of $\Gamma$. Then, for $p \in[1, \infty), \Xi$ is an $S_{p}$-set if and only if both $\Xi_{1}$ and $\Xi_{2}$ are $S_{p}$-sets.

(b) Let $p \in[1, \infty)$ and suppose $\Xi_{1}$ is an $S_{p}$-set and $\Xi_{2}$ is a $C_{p}$-set. Then $\Xi=\Xi_{1} \cup \Xi_{2}$ is an $S_{p}$-set.

The final result of this section gives us an inclusion result between the set of $C_{p}$-sets (respectively $S_{p}$-sets) and the set of $C_{q}$-sets (respectively $S_{q}$-sets) for $1 \leqq p<q \leqq \infty$.

THEOREM 1.5. Let $1 \leqq p<q \leqq \infty$. Then every $C_{p}$-set (respectively $S_{p}$-set) is a $C_{q}$-set (respectively $S_{q}$-set). 
Proof. Assume $\Xi$ is a $C_{p}$-set. Suppose we are given $\epsilon>0$ and $f \in L^{1} \cap C_{0}(G)$ with $\operatorname{supp}(\hat{f})$ compact and $\hat{f}$ vanishing on $\Xi$. We can find $h \in L^{\prime} \cap C_{0}(G)$ such that $\|f-h * f\|_{q}<\epsilon / 2$. Since $\Xi$ is a $C_{p}$-set there exists $g \in L^{\prime}(G)$ such that $\hat{g}$ has compact support disjoint from $\Xi$ and $\|h\|_{r}\|f-g * f\|_{p}<\epsilon / 2$, where $p^{-1}+r^{-1}-q^{-1}=1$ (with the usual convention for the cases $p=1$ and $q=\infty$ ). Now (see [1], (20.18))

$$
\begin{aligned}
\|f-h * g * f\|_{q} & \leqq\|f-h * f\|_{q}+\|h\|_{r}\|f-g * f\|_{p} \\
& <\epsilon .
\end{aligned}
$$

It remains only to note that $h * g \in L^{1} \cap C_{0}(G)$ and $(h * g)^{\wedge}$ has compact support disjoint from $\Xi$.

The proof that every $S_{p}$-set is an $S_{q}$-set is similar.

\section{Examples of $S_{p^{-}}$and $C_{p}$-sets.}

THEOREM 2.1. For $p \in[2, \infty]$ every closed subset of $\Gamma$ is a $C_{p}$-set.

Proof. In view of Theorem 1.5 we need only prove the theorem for $p=2$.

Let $\Xi$ be a closed subset of $\Gamma$ and suppose we are given $\epsilon>0$ and $f \in L^{1} \cap C_{0}(G)$ with $\operatorname{supp}(\hat{f})$ compact, $\hat{f}$ vanishing on $\Xi$ and $\|f\|_{1} \leqq$ 1. Now $\Omega=\{\gamma \in \Gamma: \hat{f}(\gamma) \neq 0\}$ is a relatively compact open set, and hence there exists a compact set $Y \subset \Omega$ such that $\theta(\Omega \mid Y)<\epsilon^{2}$. Choose an open set $\nabla$ such that $Y \subset \nabla \subset \nabla^{-} \subset \Omega$, and (see [3], 2.6.1) $k \in L^{1} \cap$ $C_{0}(G)$ such that $\xi_{Y} \leqq \hat{k} \leqq \xi_{\nabla}$. Then, using Plancherel's theorem,

$$
\begin{aligned}
\|f-k * f\|_{2} & =\left(\int_{\Omega \mid Y}|1-\hat{k}(\gamma)|^{2}|\hat{f}(\gamma)|^{2} d \theta(\gamma)\right)^{\frac{1}{2}} \\
& <\theta(\Omega \mid Y)^{\frac{1}{2}} \\
& <\epsilon ;
\end{aligned}
$$

and clearly, $\hat{k}$ has compact support disjoint from $\Xi$.

Definition 2.2. Let $\Omega$ be a relatively compact open subset of $\Gamma$. We shall call $\Omega$ a $\beta$-symmetry set $(\beta>0)$ if there exist nets $\left\{Y_{i}\right\}_{i \in I}$ and $\left\{\nabla_{i}\right\}_{i \in I}$ such that each $Y_{i}$ is compact, $\left\{\nabla_{i}\right\}_{i \in I}$ is a base of symmetric open neighbourhoods of zero in $\Gamma$, partially ordered by

$$
\nabla_{i}<\nabla_{j} \text { if and only if } \nabla_{i} \supset \nabla_{j}
$$

$\left(Y_{i}+2 \nabla_{i}\right)^{-} \subset \Omega$ for each $i \in I$, and

$$
\lim _{i \in I} \frac{\theta\left(\Omega \mid Y_{i}\right)^{\beta}}{\theta\left(\nabla_{i}\right)}=0
$$


TheOREM 2.3. Suppose we are given $\beta>0$ and a closed subset $\Xi$ of $\Gamma$ with the property that for any relatively compact set $Y \subset \Xi^{c}$ there exists a $\beta$-symmetry set $\Omega$ such that $Y \subset \Omega \subset \Xi^{c}$. Then $\Xi$ is a $C_{p}$-set for all $p \geqq(2+\beta)^{-1}(2+2 \beta)$.

Proof. Let $p=(2+\beta)^{-1}(2+2 \beta)$. Suppose we are given $\epsilon>0$ and $f \in L^{\prime} \cap C_{0}(G)$, where $\operatorname{supp}(\hat{f})$ is compact, $\hat{f}$ vanishes on $\Xi$ and $\|f\|_{1} \leqq 1$. Now $Y=\{\gamma \in \Gamma: \hat{f}(\gamma) \neq 0\}$ is a relatively compact open subset of $\Xi^{c}$ and hence, by assumption, there exists a relatively compact open set $\Omega$ such that $Y \subset \Omega \subset \Xi^{c}$, and nets $\left\{Y_{i}\right\}_{i \in I}$ and $\left\{\nabla_{i}\right\}_{i \in I}$ satisfying the conditions of Definition 2.2. Choose $i \in I$ such that $Y_{\text {, }}$ is nonvoid and

$$
\left[\frac{\theta\left(\Omega \mid Y_{i}\right)^{\beta}}{\theta\left(\nabla_{i}\right)}\right]^{\alpha / 2}<2^{-\alpha} \theta(\Omega)^{-\alpha / 2} \epsilon
$$

where $\alpha=(1+\beta)^{-1}$. Define $k_{i}=\theta\left(\nabla_{1}\right)^{-1} g_{i} h_{i}$, where $g_{i}, h_{i}$ in $L^{2}(G)$ are such that $\hat{g}_{i}=\xi_{\nabla_{i}}$ (cf. [3], 2.6.1) $k_{i} \in L^{\prime} \cap C_{0}(G), \xi_{Y_{i}} \leqq \hat{k}_{i} \leqq \xi_{Y_{i}+2 \nabla_{i}}$ and

$$
\left\|k_{i}\right\|_{1} \leqq\left[\frac{\theta\left(\Upsilon_{i}+\nabla_{i}\right)}{\theta\left(\nabla_{i}\right)}\right]^{\frac{1}{2}} .
$$

It follows from Hölder's inequality that

$$
\begin{aligned}
\left\|f-k_{i} * f\right\|_{p} & \leqq\left\|f-k_{i} * f\right\|_{1}^{\alpha}\left\|f-k_{i} * f\right\|_{2}^{1-\alpha} \\
& \leqq\|f\|_{1}^{\alpha}\left[1+\left[\frac{\theta\left(Y_{i}+\nabla_{i}\right)}{\theta\left(\nabla_{i}\right)}\right]^{\frac{1}{2}}\right]^{\alpha} \theta\left(\Omega \mid Y_{i}\right)^{(1-\alpha) / 2} \\
& \leqq 2^{\alpha} \theta\left(Y_{i}+\nabla_{i}\right)^{\alpha / 2} \frac{\theta\left(\Omega \mid Y_{i}\right)^{(1-\alpha) / 2}}{\theta\left(\nabla_{i}\right)^{\alpha / 2}} \\
& <\epsilon
\end{aligned}
$$

(recall that $\alpha=(1+\beta)^{-1}$ and $\left.p=(2+\beta)^{-1}(2+2 \beta)=2\left(1+\alpha^{-1}\right)^{-1}\right)$. Noting that $\hat{k}_{i}$ has compact support disjoint from $\Xi$ we see that $\Xi$ is a $C_{p}$-set, and the conclusion follows from Theorem 1.5.

We have two corollaries when $G$ is a Euclidean space.

CoROllary 2.4. Let $m \geqq 1$ and suppose $\Xi \subset R^{m}$ is an open set with the property that for any relatively compact set $Y \subset R^{m}$ there exists a number $\kappa_{m}\left(=\kappa_{m}(Y)\right)$ such that

$$
\theta\left((\partial(\Xi) \cap Y)+\nabla_{n}\right) \leqq \kappa_{m} n^{-1}
$$


for all $n \in\{1,2, \cdots\}$, where $\partial(\Xi)$ denotes the boundary of $\Xi$ and

$$
\nabla_{n}=\left\{x \in R^{m}:\|x\|<n^{-1}\right\} .
$$

Then $\Xi, \Xi^{c}$ and $\partial(\Xi)$ are $C_{p}$-sets for all $p>(2+m)^{-1}(2+2 m)$.

Proof. By Theorem 1.3 (c) we need consider only $\partial(\Xi)$.

Let $Y$ be any relatively compact open subset of $\partial(\Xi)^{c}$. We shall show that for any $\epsilon>0$ there exists an $(m+\epsilon)$-symmetry set $\Omega$ such that $Y \subset \Omega \subset \partial(\Xi)^{c}$. Since $Y$ is relatively compact in $R^{m}$ there exists an integer $n_{0}>0$ such that

$$
\Upsilon \subset \Delta_{n_{0}}=\left\{x \in R^{m}:\|x\|<n_{0}\right\} .
$$

For each $n \in\{1,2, \cdots\}$ define

$$
Y_{n}=\left(\partial(\Xi)+\nabla_{n}\right)^{c} \cap\left(\Delta_{n 0} \mid \Delta_{n 0^{-} n^{-1}}^{-1}\right)^{c} \cap \Delta_{n 0^{\prime}} .
$$

Clearly $Y_{n}$ is compact and

$$
\left(\Upsilon_{n}+2 \nabla_{3 n}\right)^{-} \subset \Delta_{n_{0}} \cap \partial(\Xi)^{c}
$$

Putting $\Omega=\Delta_{n_{0}} \cap \partial(\Xi)^{c}$ we have

$$
\begin{aligned}
\Omega \mid Y_{n} & =\left(\Omega \cap\left(\partial(\Xi)+\nabla_{n}\right)\right) \cup\left(\Omega \cap\left(\Delta_{n 0} \mid \Delta_{n_{0}-n^{-1}}^{-}\right)\right) \\
& =\left(\Delta_{n 0} \cap \partial(\Xi)^{c} \cap\left(\partial(\Xi)+\nabla_{n}\right)\right) \cup\left(\Delta_{n 0} \cap \partial(\Xi)^{c} \cap\left(\Delta_{n 0} \mid \Delta_{n_{0}-n^{-1}}^{-}\right)\right) \\
& \subset\left(\Delta_{n_{0}} \cap\left(\partial(\Xi)+\nabla_{n}\right)\right) \cup\left(\Delta_{n_{0}} \mid \Delta_{n_{0}-n^{-1}}^{-}\right) \\
& \subset\left(\left(\left(\Delta_{n 0}+\nabla_{1}\right) \cap \partial(\Xi)\right)+\nabla_{n}\right) \cup\left(\Delta_{n_{0}} \mid \Delta_{n_{0}-n^{-1}}^{-}\right) .
\end{aligned}
$$

Hence, since $\Delta_{n_{0}}+\nabla_{1}$ is relatively compact,

$$
\theta\left(\Omega \backslash Y_{n}\right) \leqq \kappa_{m}\left(\Delta_{n_{0}}+\nabla_{1}\right) n^{-1}+O\left(n^{-1}\right) .
$$

Using the fact that

$$
\theta\left(\nabla_{3 n}\right)=\kappa_{m}^{\prime} 3^{-m} n^{-m}
$$

for some constant $\kappa_{m}^{\prime}$, we have

$$
\lim _{n \rightarrow \infty} \frac{\theta\left(\Omega \mid Y_{n}\right)^{m+\epsilon}}{\theta\left(\nabla_{3 n}\right)}=0
$$

and so $\Omega$ is an $(m+\epsilon)$-symmetry set for all $\epsilon>0$. 
Thus $\partial(\Xi)$ satisfies the conditions of Theorem 2.3 with $\beta=m+\epsilon$, and hence is a $C_{p}$-set for ah $p>(2+m)^{-1}(2+2 m)$.

Corollary 2.5. Let $m \geqq 1$ and put

$$
\Xi=\left\{x \in R^{m}:\|x\|=1\right\} .
$$

Then $\Xi$ is a $C_{p}$-set for all $p>(2+m)^{-1}(2+2 m)$.

Proof. Let $\nabla$ be any relatively compact set in $R^{m}$. Then

$$
\begin{aligned}
\theta\left((\Xi \cap \nabla)+\nabla_{n}\right) & \leqq \theta\left(\Xi+\nabla_{n}\right) \\
& =\kappa_{m}^{\prime}\left(\left(1+n^{-1}\right)^{m}-\left(1-n^{-1}\right)^{m}\right) \\
& =O\left(n^{-1}\right),
\end{aligned}
$$

where $\kappa_{m}^{\prime}$ is a constant. Now apply Corollary 2.4.

REMARK 2.6. For $m \geqq 3$, Corollary 2.5 gives an example of a $C_{p}$-set $\left((2+m)^{-1}(2+2 m)<p<2\right)$ which is not an $S$-set; cf. [3], 7.3.2.

3. The failure of certain closed sets to be $S_{p}$-sets. In this section we use a proof along the lines of that of Malliavin's theorem ([3], 7.6.1) to show that every nondiscrete $\Gamma$ contains a closed set which is not an $S_{p}$-set for any $p \in[1,2)$. As in the proof of [3], Theorem 7.6.1, we first consider the cases:

(a) $\Gamma$ is an infinite compact group;

(b) $\Gamma=R$.

THEOREM 3.1. Let $G$ be an infinite discrete group. Then there exists a closed set $\Xi \subset \Gamma$ which is not an $S_{p}$-set for any $p \in[1,2)$.

Proof. Using the notation of [3], Theorem 7.8.6 we consider the function $\phi_{1}$ on $G$ defined by

$$
\phi_{1}: x \rightarrow\left(D^{1} m_{x}\right)(\zeta)
$$

It is easily proved from [3], 7.6.4 and Theorem 7.8.6 that $f_{0} \in L^{\prime}(G)$ and $\phi_{1}$ (as above) can be chosen so that $f_{0}$ and $\zeta$ satisfy the hypotheses of [3], 7.6.3 (Theorem) (with $f=f_{0}$ and $\xi=\zeta$ ) and $\phi_{1} \in L^{a}(G)$ for all $q>2$. Having thus chosen $f_{0}$ and $\phi_{1}$ we shall prove that the closed set $\Xi=\left\{\gamma \in \Gamma: \hat{f}_{0}(\gamma)=\zeta\right\}$ is not an $S_{p}$-set for any $p \in[1,2)$. 
Let $p \in[1,2)$ and put

$$
\begin{aligned}
I & =\left\{f \in L^{\prime}(G): \hat{f}(\Xi)=\{0\}\right\}, \\
I_{1} & =\text { the closed ideal of } L^{\prime}(G) \text { generated by } f_{0}-\zeta \xi_{\{0\}}, \\
I_{2} & =\text { the closed ideal of } L^{\prime}(G) \text { generated by }\left(f_{0}-\zeta \xi_{\{0\}}\right)^{* 2},
\end{aligned}
$$

and $\quad J=\left\{f \in L^{\prime}(G): \hat{f} \text { vanishes on a neighbourhood of } \Xi\right\}^{-}$.

Clearly

$$
\Xi=Z(I)=Z\left(I_{1}\right)=Z\left(I_{2}\right)=Z(J)
$$

(where $Z(I)$ denotes the zero set of the ideal $I$; see [3], 7.1.3). Since $I$ and $J$ are respectively the largest and smallest closed ideals in $L^{1}(G)$ having $\Xi$ as their zero set, we have that $J \subset I_{2} \subset I_{1} \subset I$.

As $\phi_{1} \in L^{p^{\prime}}(G)$ we can define a continuous linear functional $T$ on $\left(L^{\prime}(G),\|\cdot\|_{p}\right)$ by

$$
T(g)=\sum_{x \in G} g(-x) \phi_{1}(x)
$$

(recall that $G$ is discrete and hence $L^{\prime}(G) \subset L^{p}(G)$ ). By [3], 7.6.3, $T$ annihilates $I_{2}$ but not $I_{1}$.

Now suppose that $\Xi$ is an $S_{p}$-set and let $h \in L^{1} \cap C_{0}(G)=L^{1}(G)$ with $\hat{h}$ vanishing on $\Xi$. Then, given $\epsilon>0$, there exists $h^{\prime} \in J$ such that $\left\|\dot{h}-h^{\prime}\right\|_{p}<\epsilon$ and hence, since $T\left(h^{\prime}\right)=0, \quad|T(h)|=\left|T\left(h-h^{\prime}\right)\right| \leqq$ $\epsilon\left\|\phi_{1}\right\|_{p^{\prime}}$. As this holds for all $\epsilon>0$ we must have that $T(h)=0$; thus $T$ annihilates $I$, a contradiction of the fact that $T$ does not annihilate $I, \subset I$. It follows that $\Xi$ is not an $S_{p}$-set for any $p \in[1,2)$.

We shall now examine the case when $\Gamma$ contains an infinite compact open subgroup. We require two lemmas for arbitrary Hausdorff locally compact Abelian groups.

Lemma 3.2. Let $G$ be a Hausdorff locally compact Abelian group and suppose $H$ is a closed subgroup of $G$. Then a continuous integrable function $f$ on $G$ is constant on cosets of $H$ if and only if

$$
\operatorname{supp}(\hat{f}) \subset A(\Gamma, H)
$$

(the annihilator of $H$ in $\Gamma$ ).

Proof. The result follows readily from the property

$$
\left({ }_{h} f\right)^{\wedge}(\gamma)=\gamma(h) \hat{f}(\gamma)
$$

for all $\gamma \in \Gamma$ (where $\left.{ }_{h} f: x \rightarrow f(x+h)\right)$. 
Lemma 3.3. Let $G$ be a Hausdorff locally compact Abelian group and suppose $\Lambda$ is an open subgroup of $\Gamma$. If $\Xi$ is a closed subset of $\Lambda$ which is not an $S_{p}$-set in $\Lambda$ then $\Xi$ is not an $S_{p}$-set in $\Gamma$.

Proof. Put $H=A(G, \Lambda)$. By [1], (23.24) (e), $H$ is compact. Furthermore, in view of Theorem 2.1, we can assume that $p<\infty$.

Suppose, to the contrary, that $\Xi$ is an $S_{p}$-set in $\Gamma$. Given $\epsilon>0$ and $\dot{f} \in L^{1} \cap C_{0}(G / H)$ such that $\operatorname{supp}(\hat{f})$ is compact and $\hat{\dot{f}}$ vanishes on $\Xi$, put $f=\dot{f} \circ \pi_{H}$, where $\pi_{H}$ denotes the natural homomorphism of $G$ onto $G / H$. Denoting the Haar measures on $H, G / H$ by $\lambda_{H}, \lambda_{G / H}$ respectively (normalised as in [2], Chapter 3, 3.3 (i) with $\lambda_{H}(H)=1$ ) we have, by [2], Chapter 3, 4.5,

$$
\begin{aligned}
\|f\|_{p}^{p} & =\int_{G / H}\left\{\int_{H}|f(x+y)|^{p} d \lambda_{H}(y)\right\} d \lambda_{G / H}(\dot{x}) \\
& =\int_{G / H}\left\{\int_{H}\left|\dot{f} \circ \pi_{H}(x+y)\right|^{p} d \lambda_{H}(y)\right\} d \lambda_{G / H}(\dot{x}) \\
& =\int_{G / H}|\dot{f}(\dot{x})|^{p} d \lambda_{G / H}(\dot{x}),
\end{aligned}
$$

that is,

$$
\|f\|_{p}=\|\dot{f}\|_{p}
$$

It is easily seen that

$$
\dot{f}(\dot{x})=\int_{H} f(x+y) d \lambda_{H}(y)
$$

and, by [2], Chapter $4,4.3$ ((3.1) shows that $\left.f \in L^{\prime}(G)\right)$,

$$
\hat{\dot{f}}(\gamma)=f(\gamma)
$$

for all $\gamma \in \Lambda$. Furthermore, since $f$ is constant on cosets of $H$, Lemma 3.2 shows that $\operatorname{supp}(\hat{f}) \subset A(\Gamma, H)=\Lambda$. As $\operatorname{supp}(\hat{\hat{f}})$ is assumed to be compact it follows from (3.2) that $\operatorname{supp}(\hat{f})$ is compact and hence (note that $f$ is continuous) we see that $f \in C_{0}(G)$.

Now $\hat{f}$ vanishes on $\Xi \cup \Lambda^{c}$ and, since by Theorem 1.4 (recall that $\Lambda^{c}$ is open and closed) $\Xi \cup \Lambda^{c}$ is an $S_{p}$ - set; there exists $g \in L^{\prime} \cap C_{0}(G)$ such that $\hat{g}$ has compact support disjoint from $\Xi \cup \Lambda^{c}$ and $\|f-g\|_{p}<\epsilon$. By Lemma 3.2 again $g$ is constant on cosets of $H$ and we have the existence of $\dot{g} \in L^{1} \cap C_{0}(G / H)$ such that $g=\dot{g} \circ \pi_{H}\left(\dot{g} \in C_{0}(G / H)\right.$ since, by [2], Chapter $3,1.8$ (vii), $\dot{g}$ is continuous and by (3.2), $\hat{g}$ has compact support). From (3.1) $\|\dot{f}-\dot{g}\|_{p}<\epsilon$, and (3.2) shows that $\dot{\dot{g}}$ vanishes on a 
neighbourhood of $\Xi$. Hence $\Xi$ is shown to be an $S_{p}$-set in $\Lambda$, contrary to assumption.

COROllary 3.4. Let $G$ be a Hausdorff locally compact Abelian group, $\Gamma$ its character group. If $\Gamma$ contains an infinite compact open subgroup then there exists a closed subset of $\Gamma$ which is not an $S_{p}$-set for any $p \in[1,2)$.

Proof. Combine Theorem 3.1 and Lemma 3.3.

Before considering the case $\Gamma=R$ we need to extend the result in [3], Theorem 2.7.6.

Theorem 3.5. Suppose $f \in l^{1}(Z), \delta \in(0, \pi)$ and $\hat{f}(\exp (i x))=0$ for $x \in[\pi-\delta, \pi+\delta]$. Let $u$ be defined on $R$ by

$$
u(x)= \begin{cases}\hat{f}(\exp (i x)) & (|x| \leqq \pi) \\ 0 & (|x|>\pi)\end{cases}
$$

Then $u=\hat{g}$ for some $g \in L^{1}(R)$. Moreover, given $p \in[1, \infty]$, there exists a positive number $\kappa_{p}\left(=\kappa_{p}(\delta)\right)$ such that

$$
\|f\|_{p} \leqq \kappa_{p}\|g\|_{p} .
$$

Proof. The first part of Theorem 3.5 is proved in [3], 2.7.6.

Let $p \in[1, \infty]$. Consider the linear operator $T$ from $L^{1} \cap L^{\infty}(R)$ to $l^{1}(Z)$, defined by

$$
(T(k))(n)=k * \hat{h}(n),
$$

where $n \in Z$, and $h \in L^{1}(R)$ is defined as in [3], 2.7.6. The argument at the end of the proof of [3], 2.7.6 shows that there is a constant $\kappa_{1}=\kappa_{1}(\delta)$ such that $\|T(k)\|_{1} \leqq \kappa_{1}\|k\|_{1}$. It is clear from (3.3) that $\|T(k)\|_{\infty} \leqq \kappa_{2}\|k\|_{\infty}$, where $\kappa_{2}=\|\hat{h}\|_{1}$. By the Riesz-Thorin convexity theorem $T$ is continuous as

$$
\left(L^{1} \cap L^{\infty}(R),\|\cdot\|_{p_{\alpha}}\right) \stackrel{T}{\rightarrow}\left(l^{1}(Z),\|\cdot\|_{p_{\alpha}}\right)
$$

(recall that $l^{\prime}(Z) \subset l^{\infty}(Z)$ ), where $\alpha \in(0,1), p_{\alpha}=(1-\alpha)^{-1}$ and $\|T\|_{(\alpha)} \leqq$ $\kappa_{1}^{1-\alpha} \kappa_{2}^{\alpha}$. In particular, choosing $\alpha \in[0,1)$ such that $p_{\alpha}=p$ (and $\alpha=1$ if $p=\infty$ ) and noting that $g \in L^{1} \cap L^{\infty}(R)$ and (see [3], 2.7.6, (5)) $f(n)=$ $g * \hat{h}(n)$ for all $n \in Z$, we have

as required.

$$
\|f\|_{p} \leqq \kappa_{1}^{1-\alpha} \kappa_{2}^{\alpha}\|g\|_{p},
$$


THEOREM 3.6. The real line $R$ contains a closed set which is not an $S_{p}$-set for any $p \in[1,2)$.

Proof. It appears from Theorem 3.1 that there exists a closed set $\Xi_{1} \subset T$ (the circle group) which is not an $S_{p}$-set for any $p \in[1,2)$. By translation if necessary we can assume that $-1 \notin \Xi_{1}$ and that $\Xi_{1}$ is disjoint from $\Xi_{2}$ for some closed arc $\Xi_{2} \subset T$ containing -1 . Put

$$
\begin{aligned}
& Y_{1}=\left\{x \in(-\pi, \pi): \exp (i x) \in \Xi_{1}\right\}, \\
& \Upsilon_{2}=\left\{x \in(-\pi, \pi): \exp (i x) \in \Xi_{2}\right\} \cup[\pi, \infty) \cup(-\infty,-\pi], \\
& \Xi=\Xi_{1} \cup \Xi_{2} \text { and } Y=\Upsilon_{1} \cup Y_{2} .
\end{aligned}
$$

Let $p \in[1,2)$ and suppose $Y_{1}$ is an $S_{p}$-set. By Theorem 1.4, $Y$ is an $S_{p}$ - set. Given $f \in l^{1}(Z)$ with $\hat{f}(\Xi)=\{0\}$ define $g \in L^{1} \cap C_{0}(R)$ by

$$
\hat{g}(x)= \begin{cases}\hat{f}(\exp (i x)) & (|x| \leqq \pi) \\ 0 & (|x|>\pi)\end{cases}
$$

(see Theorem 3.5). Clearly $\hat{g}$ vanishes on $Y$ and hence, since $Y$ is an $S_{p}$-set, there exists a sequence $\left(g_{n}\right) \subset L^{1} \cap C_{0}(R)$ such that each $\hat{g}_{n}$ vanishes on a neighbourhood of $Y$ and

$$
\left\|g-g_{n}\right\|_{p} \rightarrow 0
$$

If, for each $x \in(-\pi, \pi]$, we define $f_{n} \in l^{1}(Z)$ by

$$
\hat{f}_{n}(\exp (i x))=\hat{g}_{n}(x)
$$

(see [3], Theorem 2.7.6) then Theorem 3.5 applied to (3.4) gives $\left\|f-f_{n}\right\|_{p} \rightarrow 0$ (note that each $\hat{f}_{n}$ vanishes on a neighbourhood of $\Xi$ ). Hence $\Xi$ and consequently (see Theorem 1.4) $\Xi_{1}$ would be an $S_{p}$-set, contradicting our choice of $\Xi_{1}$. It follows that $Y_{1}$ is not an $S_{p}$-set for any $p \in[1,2)$.

We require two lemmas before proving the main result of this section.

Lemma 3.7. Let $G, H$ be Hausdorff locally compact Abelian groups and suppose $k \in L^{1} \cap C_{0}(G \times H)$ is such that $Y=\operatorname{supp}(\hat{k})$ is compact. Then the function $y \rightarrow k(x, y)(x \rightarrow k(x, y))$ is integrable over 
$H$ for every $x \in G$ (over $G$ for every $y \in H$ ). Furthermore the functions

$$
\phi_{1}: x \rightarrow \int_{H} k(x, y) d \lambda_{H}(y), \quad \phi_{2}: y \rightarrow \int_{G} k(x, y) d \lambda_{G}(x)
$$

are continuous.

Proof. Since $k$ is continuous the function $y \rightarrow k(x, y)$ is continuous, and hence measurable, for every $x \in G$.

Choose $k_{1}\left(k_{2}\right)$ in $L^{1} \cap C_{0}(G)\left(L^{1} \cap C_{0}(H)\right)$ such that $\hat{k}_{1}=1\left(\hat{k}_{2}=1\right)$ on a neighbourhood $\nabla_{1}\left(\nabla_{2}\right)$ of $Y_{G}\left(\Upsilon_{H}\right)$, where $\Upsilon_{G}, \Upsilon_{H}$ are the projections of $\Upsilon$ onto $G, H$ respectively. If we define $h$ on $G \times H$ by $h[(x, y)]=$ $k_{1}(x) k_{2}(y)$ then [1], (31.7) (b) shows that $\hat{h}=1$ on $\nabla_{1} \times \nabla_{2}$, a neighbourhood of $Y$. Thus $h * k=k$ 1.a.e. and, since $h * k$ and $k$ are continuous,

$$
h * k=k .
$$

Now the map $\nu_{x}$ on $H \times G \times H$, defined by

$$
\nu_{x}[(y, s, t)]=h(x-s, y-t) k(s, t),
$$

is continuous for every $x \in G$. Applying [1], (13.4) to $\left|\nu_{x}\right|$, considered as a function on $H \times(G \times H)$, it follows that $\nu_{x}$ is integrable and, using (3.5), that the function $y \rightarrow k(x, y)$ is integrable over $H$ for every $x \in G$. Furthermore, since $\nu_{x}$ is integrable on $H \times(G \times H)$, we can use (3.5) and [1], (13.8) to deduce that

$$
\phi_{1}(x)=\int_{H} k_{2}(y) d \lambda_{H}(y) \int_{G \times H} k_{1}(x-s) k(s, t) d \lambda_{G} \times \lambda_{H}(s, t) .
$$

As $k \in L^{1}(G \times H), k_{2} \in L^{1}(H)$ and $k_{1}$ is uniformly continuous it follows that $\phi_{1}$ is continuous.

The other part of the lemma is proved similarly.

Lemma 3.8. Suppose $G, H$ are Hausdorff locally compact Abelian groups, with character groups $\Gamma, \Lambda$ respectively. If $p \in[1,2)$ and the closed set $\Xi^{\prime} \subset \Gamma$ is not an $S_{p}$-set, then $\Xi=\Xi^{\prime} \times \Lambda$ is not an $S_{p}$-set in $\Gamma \times \Lambda$.

Proof. Suppose to the contrary that $\Xi$ is an $S_{p}$-set in $\Gamma \times \Lambda$. Let $f \in L^{1} \cap C_{0}(G)$ with $\operatorname{supp}(\hat{f})$ compact and $\hat{f}$ vanishing on $\Xi$, and choose $g \in L^{1} \cap C_{0}(H)$ such that $\operatorname{supp}(\hat{g})$ is compact and $|g(y)| \geqq 1$ for all $y$ in 
some neighbourhood $V$ of zero in $H$. Define $h$ on $G \times H$ by $h[(x, y)]=f(x) g(y)$. Then, by [1], (31.7) (b), $\operatorname{supp}(\hat{h})$ is compact and

$$
\hat{h}\left(\left[\gamma_{1}, \gamma_{2}\right]\right)=\hat{f}\left(\gamma_{1}\right) \hat{g}\left(\gamma_{2}\right)=0
$$

for all $\left[\gamma_{1}, \gamma_{2}\right] \in \Xi$.

Let $\epsilon>0$ be given. Since $\Xi$ is assumed to be an $S_{p}$-set we can find $k \in L^{1} \cap C_{0}(G \times H)$ such that $\operatorname{supp}(\hat{k})$ is compact and disjoint from $\Xi$, and

$$
\|h-k\|_{p}<\epsilon \lambda_{H}(V)^{1 / p}
$$

Thus, for all $\gamma_{1}$ in some neighbourhood $\nabla$ of $\Xi^{\prime}$ and for all $\gamma_{2} \in \Lambda$, we have (see [1], (13.8))

$$
\begin{aligned}
& \int_{H}\left\{\int_{G} k(x, y) \bar{\gamma}_{1}(x) d \lambda_{G}(x)\right\} \bar{\gamma}_{2}(y) d \lambda_{H}(y) \\
& \quad=\int_{G \times H} k(x, y)\left(\left[\gamma_{1}, \gamma_{2}\right]\right)^{-}(x, y) d \lambda_{G} \times \lambda_{H}(x, y) \\
& \quad=0 .
\end{aligned}
$$

Since $\gamma_{2} \in \Lambda$ was chosen arbitrarily

$$
\int_{G} k(x, y) \bar{\gamma}_{1}(x) d \lambda_{G}(x)=0 \quad \lambda_{H} \text {-a.e. }
$$

Now

$$
\psi:(x, y) \rightarrow k(x, y) \bar{\gamma}_{1}(x)
$$

is continuous and integrable, and $\operatorname{supp}(\hat{\psi})$ is compact. Hence, by Lemma 3.7, the function $\phi$ on $H$ defined by

$$
\phi(y)=\int_{G} \psi(x, y) d \lambda_{G}(x)
$$

is continuous and so, for all $y \in H$ and $\gamma_{1} \in \nabla$,

$$
\int_{G} k(x, y) \bar{\gamma}_{1}(x) d \lambda_{G}(x)=0 .
$$

Using (3.6) we see that

$$
W=\left\{y \in V: \int_{G}|h(x, y)-k(x, y)|^{p} d \lambda_{G}(x)<\epsilon^{p}\right\}
$$


has the property that $\lambda_{H}(V \backslash W)<\lambda_{H}(V)$, that is, $\lambda_{H}(W)>0$. Choose any $y_{0} \in W$ ( $W$ is nonempty). Then

$$
\int_{G}\left|f(x)-g\left(y_{0}\right)^{-1} k\left(x, y_{0}\right)\right|^{p} d \lambda_{G}(x)<\epsilon^{p}\left|g\left(y_{0}\right)\right|^{-1} \leqq \epsilon^{p}
$$

and so, defining $f_{1} \in L^{1} \cap C_{0}(G)$ by $f_{1}(x)=g\left(y_{0}\right)^{-1} k\left(x, y_{0}\right)$, (3.7) shows that $\hat{f}_{1}$ vanishes on $\nabla$ and, from (3.8), $\left\|f-f_{1}\right\|_{p}<\epsilon$; thus we have a contradiction of the assumption that $\Xi^{\prime}$ is not an $S_{p}$-set.

THEOREM 3.9. Let $G$ be a Hausdorff noncompact locally compact Abelian group, $\Gamma$ its character group. Then $\Gamma$ contains a closed set which is not an $S_{p}$-set for any $p \in[1,2)$.

Proof. By [1], (24.30), $\Gamma$ is topologically isomorphic with $R^{n} \times \Gamma_{0}$, where $\Gamma_{0}$ is a Hausdorff locally compact Abelian group containing a compact open subgroup.

If $n \geqq 1$ then Theorem 3.6 and Lemma 3.8 combine to show that $R^{n} \times \Gamma_{0}$ contains a closed set which is not an $S_{p}$-set for any $p \in[1,2)$.

If $n=0$ then $\Gamma$ contains a compact open subgroup (with is infinite since $\Gamma$ is nondiscrete) and the result follows from Corollary 3.4.

\section{REFERENCES}

1. Edwin Hewitt and Kenneth A. Ross, Abstract Harmonic Analysis, vols. I, II, Die Grundlehren der mathematischen Wissenschaften, Bände 115, 152. Academic Press, New York; SpringerVerlag, Berlin, Göttingen, Heidelberg, 1963, 1970.

2. Hans Reiter, Classical Harmonic Analysis and Locally Compact Groups, Oxford at the Clarendon Press, 1968.

3. Walter Rudin, Fourier Analysis on Groups, Interscience Publishers, John Wiley and Sons, New York, London, 1962; 2nd printing, 1967.

Received September 11, 1974.

The Australian National University, Canberra, ACT, Australia

Present address: Murdoch University

Murdoch, Western Australia, Australia 

D. E. Bennett, Strongly unicoherent continua ............................. 1

Walter R. Bloom, Sets of p-spectral synthesis ................................ 7

R. T. Bumby and D. E. Dobbs, Amitsur cohomology of quadratic extensions: Formulas and number-theoretic examples ................. 21

W. W. Comfort, Compactness-like properties for generalized weak topological sums

D. R. Dunninger and J. Locker, Monotone operators and nonlinear biharmonic boundary value problems ...

T. S. Erickson, W. S. Martindale, 3rd and J. M. Osborn, Prime nonassociative algebras

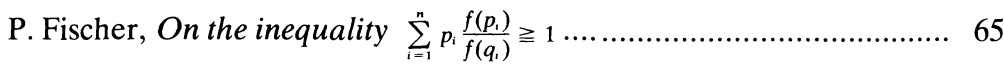

G. Fox and P. Morales, Compact subsets of a Tychonoff set ............... 75

R. Gilmer and J. F. Hoffmann, A characterization of Prüfer domains in terms of polynomials ......................................................... 81

L. C. Glaser, On tame Cantor sets in spheres having the same projection in each direction ......................................................... 87

Z. Goseki, On semigroups in which $X=X Y X=X Z X$ if and only if

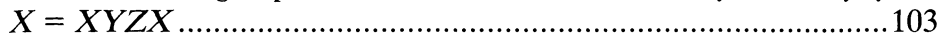

E. Grosswald, Rational valued series of exponentials and divisor

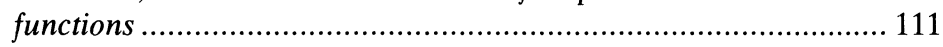

D. Handelman, Strongly semiprime rings ...................................... 115

J. N. Henry and D. C. Taylor, The $\bar{\beta}$ topology for $w^{*}$-algebras ............. 123

M. J. Hodel, Enumeration of weighted p-line arrays .......................... 141

S. K. Jain and S. Singh, Rings with quasiprojective left ideals .............. 169

S. Jeyaratnam, The diophantine equation $Y(Y+m)(Y+2 m) \times$

$$
(Y+3 m)=2 X(X+m)(X+2 m)(X+3 m) \ldots \ldots \ldots \ldots \ldots \ldots \ldots . . .183
$$

$\mathrm{R}$. Kane, On loop spaces without $p$ torsion .........................................189

Alvin J. Kay, Nonlinear integral equations and product integrals ..........203

A. S. Kechris, Countable ordinals and the analytic hierarchy, I ...........223

Ka-Sing Lau, A representation theorem for isometries of $C(X, E) \ldots \ldots . .229$

I. Madsen, On the action of the Dyer-Lashof algebra in $H_{*}(G)$..........235

R. C. Metzler, Positive linear functions, integration, and Choquet's theorem ........................................................................................ 277

A. Nobile, Some properties of the Nash blowing-up ............................297

G. E. Petersen and G. V. Welland, Plessner's theorem for Riesz conjugates 


\section{Pacific Journal of Mathematics}

\section{Vol. 60, No. $1 \quad$ September, 1975}

Donald Earl Bennett, Strongly unicoherent continua ................ 1

Walter Russell Bloom, Sets of p-spectral synthesis ................ 7

Richard Thomas Bumby and David Earl Dobbs, Amitsur cohomology of

quadratic extensions: formulas and number-theoretic examples .......

W. Wistar (William) Comfort, Compactness-like properties for generalized

weak topological sums ...................................

Dennis Robert Dunninger and John Stewart Locker, Monotone operators

and nonlinear biharmonic boundary value problems ..............

Theodore Erickson, Wallace Smith Martindale, III and J. Marshall Osborn,

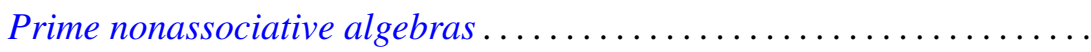

Pál Fischer, On the inequality $\sum_{i=0}^{n}\left[f\left(p_{i}\right) / f\left(q_{i}\right)\right] p_{i} \geq i \ldots \ldots \ldots \ldots \ldots$

Geoffrey Fox and Pedro Morales, Compact subsets of a Tychonoff set.......

Robert William Gilmer, Jr. and Joseph F. Hoffmann, A characterization of

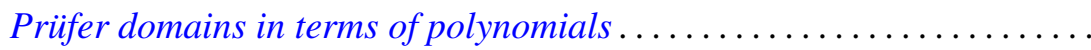

Leslie C. Glaser, On tame Cantor sets in spheres having the same projection

in each direction . ...................................

Zensiro Goseki, On semigroups in which $x=x y x=x z x$ if and only if

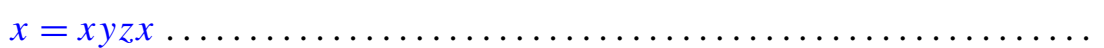

Emil Grosswald, Rational valued series of exponentials and divisor

functions.

David E. Handelman, Strongly semiprime rings

Jackson Neal Henry and Donald Curtis Taylor, The $\bar{\beta}$ topology for

$W^{*}$-algebras

Margaret Jones Hodel, Enumeration of weighted p-line arrays ...

Surender Kumar Jain and Surjeet Singh, Rings with quasi-projective left

ideals.

S. Jeyaratnam, The Diophantine equation

$$
Y(Y+m)(Y+2 m)(Y+3 m)=2 X(X+m)(X+2 m)(X+3 m) \ldots
$$

Richard Michael Kane, On loop spaces without $p$ torsion

Alvin John Kay, Nonlinear integral equations and product integrals ...

Alexander S. Kechris, Countable ordinals and the analytical hierarchy.

$I$.

Ka-Sing Lau, A representation theorem for isometries of $C(X, E)$

Ib Henning Madsen, On the action of the Dyer-Lashof algebra in $H_{*}(G)$

Richard C. Metzler, Positive linear functions, integration, and Choquet's

theorem.

Augusto Nobile, Some properties of the Nash blowing-up

Gerald E. Peterson and Grant Welland, Plessner's theorem for Riesz. 